\title{
Annexin A2 Promotes Angiogenesis After Ischemic Stroke Via Annexin A2 Receptor - AKT/ERK Pathways
}

\section{Wenlu Li}

Zhejiang University School of Medicine Second Affiliated Hospital Haoran Lin

Zhejiang University School of Medicine Second Affiliated Hospital

\section{Zexu Shen}

Zhejiang University School of Medicine Second Affiliated Hospital

\section{Yun Bei}

Zhejiang University School of Medicine Second Affiliated Hospital

\section{Taofeng Wei}

Zhejiang University School of Medicine Second Affiliated Hospital

\section{Zhanyang Yu}

Massachusetts General Hospital

\section{Yunjian Dai}

Zhejiang University School of Medicine Second Affiliated Hospital

\section{Haibin Dai ( $\nabla$ haibindai@zju.edu.cn )}

Second Affiliated Hospital, Zhejiang University School of Medicine https://orcid.org/0000-0002-57682714

\section{Research Article}

Keywords: Stroke, angiogenesis, endothelial cells, Annexin A2

Posted Date: September 24th, 2021

DOl: https://doi.org/10.21203/rs.3.rs-913984/v1

License: (c) (i) This work is licensed under a Creative Commons Attribution 4.0 International License. Read Full License 


\section{Abstract}

Promoting angiogenesis to restore circulation to the ischemic tissue is still an important therapeutic target in stroke. Here, we ask whether the $\mathrm{Ca}^{2+}$-regulated, phospholipid-and membrane-binding proteinAnnexin A2 (ANXA2) may regulate angiogenesis after stroke.Compared with wild type (WT) mice, the density of microvessels in brain and the number of new vessels sprouting from aortic ring were significantly increased in Anxa2 knock-in $\left(A N X A 2^{+/+}\right)$mice. After focal cerebral ischemia, proliferation of brain endothelial cells in $A N X A 2^{+/+}$mice was significantly elevated at 7 days post-stroke, which further improved behavioral recovery. To assess the pro-angiogenic mechanisms of ANXA2, we used brain endothelial cells cultures to investigate its effects on cell tube-formation and migration. Recombinant ANXA2 increased tube-formation and migration of brain endothelial cells either under normal condition or after OGD injury. These protective effects of recombinant ANXA2 were regulated by interaction with ANXA2 receptor (A2R), and the ability of ANXA2-A2R to activate AKT/ERK pathways. Taken together, our study indicates that ANXA2 might be involved in angiogenesis after ischemic stroke. Further investigation of ANXA2-A2R will provide a new therapeutic target for stroke.

\section{Introduction}

Stroke, as a devastating central neuronal system disease, affects over 10 million people all over the world and is one of the most common cause in cardiovascular diseases-induced death [1,2]. Prevent neuronal death and improve recovery was the priority in the past decades. However, clinically effective therapies remain elusive. Thus, to restore neurological deficits, developing novel therapies after stroke beyond the hyper-acute phase ischemia is extremely important.

As the first responder to ischemia and reperfusion injury, vasculature is the basis for any neuroprotective therapy to increase blood flow for brain energy demand, which further emphasizes the significant effect of angiogenesis in stroke recovery. Therapeutic angiogenesis is a process that pharmacologically stimulates of the regrowth of new blood vessels so as to increase cerebral blood flow to the ischemic tissue [3]. By binding to their membrane receptors on brain endothelial cells, angiogenic growth factors could regulate angiogenesis via promoting endothelial cell migration, proliferation and survival in the ischemic brain area [4].

The $\mathrm{Ca}^{2+}$-regulated, phospholipid-and membrane-binding protein, Annexin A2 (ANXA2) has been found in endothelial cells [5], tumor cells [6], monocytes, macrophages and dendritic cells [7-9]. In the oxygeninduced retinopathy (OIR), ANXA2 might promote retinal neoangiogenesis by increasing perivascular fibrin clearance through stabilizing its binding partner, p11[10]. More importantly, low dose tPA plus recombinant ANXA2 therapy significantly augmented microvessel density, synaptophysin and VEGF expression in peri-infarct areas in rat focal stroke [11]. Thus, ANXA2 is emerging as a potential regulator of angiogenesis in ischemic stroke.

\section{Methods}




\section{Animals}

Anxa2 knock-in mice ( $A N X A 2^{+/+}, \mathrm{C} 57 \mathrm{BL} / 6$ background), which overexpress $A N X A 2$, were commercially generated by standard homologous recombination at Cyagen (Suzhou, China). Male adult C57BL/6 mice (8-10 weeks) (from Shanghai Slac Laboratory Animal) were used. The environmental conditions which maintain the mouse were: 12 -hour dark/12-hour light cycle, humidity range of 30-50\%, temperature range of $20-25^{\circ} \mathrm{C}$. All behavior experiments were performed between 9:00 and 19:00. All animal experiments were conducted in accordance with the ethical guidelines of the Zhejiang University Animal Experimentation Committee and Institutional Animal Care. Every Effort has been made to minimize animal suffering, and a minimum number of animals were used.

There are three special regions on $A N X A 2^{+/+}$mice knock-in allele: region 1 is located at the border between homology arm and CAG promoter sequences; region 2 is located at the border between CAG promoter and Anxa2-cDNA sequences; region 3 is located the border between Anxa2-cDNA and homology arm sequences (Supplementary Fig. 1A). For genotyping of $A N X A 2^{+/+}$mice, four pairs of primers were applied. The primers sequences are listed in Supplementary Table. For primer pair of ANXA2-region $1 \mathrm{~F}$ and $A N X A 2$-region 1R, the PCR products were $463 \mathrm{bp}$ (region 1 of the knock-in allele). For primer pair of $A N X A 2$-region $2 \mathrm{~F}$ and $A N X A 2$-region $2 \mathrm{R}$, the $\mathrm{PCR}$ products were $383 \mathrm{bp}$ (region 2 of the knock-in allele). For primer pair of $A N X A 2$-region $3 \mathrm{~F}$ and $A N X A 2$-region $3 \mathrm{R}$, the PCR products were $437 \mathrm{bp}$ (region 3 of the knock-in allele). For the pair of Internal-control $\mathrm{F}$ and Internal-control R the PCR products were $689 \mathrm{bp}$ (Internal control) (Supplementary Fig. 1B). The PCR program was $94^{\circ} \mathrm{C}$ for 2 mins $(1 \times) ; 94^{\circ} \mathrm{C}$

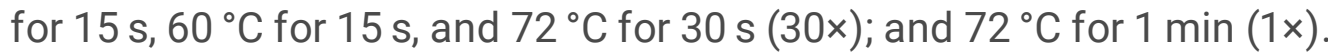

\section{Reagents}

Growth Factor Reduced Matrigel was purchased from Corning (356230, USA). Recombinant human ANXA2 protein was purchased from R\&D System (D9409-AN-050, USA). Anti-BrdU antibody was purchased from Abcam (ab1893, USA). Anti-Lectin was purchased from Vectorlabs, (B-1415-2, USA). AntiTubulin monoclonal antibody was purchased from Abcam (ab210797, USA). Anti-A2R polyclonal antibody was purchased from Novus Biologicals (NBP-53077, USA). Anti-AKT polyclonal antibody was purchased from Cell Signaling Technology (9272s, USA). Anti-p-AKT polyclonal antibody was purchased from Cell Signaling Technology (4060s, USA). Anti-ERK monoclonal antibody was purchased from Santa Cruz Biotechnology (sc-81457, USA). Anti-phospho-ERK monoclonal antibody was purchased from R\&D System (DYC1483-5, USA). Alexa Fluor ${ }^{\circledR} 488$ AffiniPure donkey anti-goat IgG were purchased from Jackson ImmunoResearch Laboratories (705-545-147, USA). Alexa Fluor® 488 IgG fraction monoclonal mouse anti-Biotin were purchased from Jackson ImmunoResearch Laboratories (200-542-211, USA). Alexa Fluor ${ }^{\circledR} 594 \mathrm{lgG}$ fraction monoclonal mouse anti-Biotin were purchased from Jackson ImmunoResearch Laboratories (200-582-211, USA).

\section{Immunohistochemistry}


Brains from mice were sliced in $20 \mu \mathrm{m}$ thickness using a Thermo Fisher ${ }^{\circledR}$ freezing microtome. After being washed 3 times with PBS for 5 mins each, the brain slices were placed into the glass Petri dish with the $10 \mathrm{mM}$ citric acid $(\mathrm{pH}=6)$ antigen retrieval buffer which was preheated to $90-100^{\circ} \mathrm{C}$. The glass Petri dish was heated in a microwave oven for 10 mins. Then it was cooled to room temperature. Next, the brain slices were permeabilized with $0.3 \%(\mathrm{w} / \mathrm{v})$ Triton X-100 in PBS at room temperature for 30 mins. After blocking in PBS containing 5\% normal donkey serum at room temperature for $2 \mathrm{~h}$, the brain slices were first incubated with anti-BrdU antibody and anti-Lectin antibody at $4{ }^{\circ} \mathrm{C}$ overnight and then with Alexa Fluor-conjugated secondary antibodies at room temperature. FluoroshieldTM with DAPI (Sigma-Aldrich, USA) was used to label the nuclei. Before permeabilizing with $0.3 \%(w / v)$ Triton X-100 in PBS, the sections used for BrdU staining were pretreated with $1 \mathrm{M}$ hydrochloric acid at $37^{\circ} \mathrm{C}$ for 30 mins before the above steps, washed with $0.1 \mathrm{M}$ sodium tetraborate for 3 times, and neutralized for 5 mins each time. Then the primary and secondary antibodies were incubated according to the above process. Images were taken by a laser confocal microscope (Leica SP8, Germany). All the image quantification analyses were done by imageJ-win64. All the data were collected and analyzed by the rater who was blind to experiments design.

\section{Aortic ring angiogenesis assay}

The assay developed by Nicosia and Ottinetti was modified [12]. Aortas of 2 weeks WT control or $A N X A 2^{+/+}$littermate mice were sliced into $1 \mathrm{~mm}$ diameter rings. Aortic rings were then added in DMEM with $2.5 \%$ fetal bovine serum (FBS) to a 24-well plate that were pre-coated with a rat tail collagen I gel (BD Biosciences, UK). Medium was changed every 2 days. At the sixth day of culture, the explants were stained by anti-Lectin (B-1415-2, Vectorlabs, USA) and visualized under a Leica SP8 laser confocal microscope.

\section{Photothrombotic model of stroke}

Photothrombotic model was performed as previously described to induce focal cerebral ischemia [13-15]. In brief, mice were anesthetized with isoflurane and the heads were fixed on a stereotaxic apparatus. A cold light source (17000 lux; diameter $2.0 \mathrm{~mm}$ ) was placed on the mouse skull surface $(1.5 \mathrm{~mm}$ lateral to bregma). Rose Bengal solution (Sigma, USA) was dissolved in saline and injected into mice intraperitoneally, at $100 \mathrm{mg} / \mathrm{kg}$. The head of the mouse was illuminated for 10 mins through the intact skull 5 mins after drug injections. Sham mouse was injected with Rose Bengal solution at same dosage and did not subject to illumination.

\section{Grid-walking test}

The grid-walking test was performed on a metal grid area of $20 \times 32 \times 50 \mathrm{~cm}$ of width, length and height, with a $12 \mathrm{~mm}$ square wire mesh, as previously described [14,15]. A video camera was placed under the apparatus to record the gait of mouse. Mouse was allowed to freely move on the apparatus for 5 mins. The number of foot errors and the normal steps of left forelimb were counted (the left forelimb was counted for 100 steps per video). The ratio of forelimb errors was calculated as follows: total number of 
foot errors / (total number of foot errors + total number of the normal steps) $* 100$. Foot errors were considered to occur when the forelimb was not providing support and the foot went through the grid hole, or animal was resting with the grid at the level of the wrist. The video analysis was performed offline by the rater who was blind to experiments design.

\section{Cylinder test}

The cylinder test was performed as previously described $[16,15]$. In short, mouse was allowed to freely explore for $5 \mathrm{~min}$ in a Plexiglas cylinder $(15 \mathrm{~cm}$ in height with a diameter of $10 \mathrm{~cm})$. A camera was positioned above the apparatus to record the exploratory rear of mouse. Exploratory rear was considered to occur when the mouse spontaneously reared by touching the wall of cylinder with its forelimbs. The time (in seconds) spent by the both forelimbs or each forelimb of mouse pressing the wall of cylinder was counted. The percentage of time spent on each forelimb were used to derive an asymmetry index which was calculated as follows: (the time spent on ipsilateral forelimb - the time spent on contralateral forelimb) / total time spend on forelimb. The video was analyzed by the rater who was blind to experiments design.

\section{Human brain microvascular endothelial cell (HBMEC) culture}

HBMECs were obtained from Cell Systems Corporation (Kirkland, WA, USA). The method of HBMECs culture has been described previously [17]. In brief, the cells of passage 6th to 12th were grown with EBM2 Basal Medium (Lonza, USA) containing Endothelial Cell Growth Medium-2 (Lonza, USA). HBMECs were cultured with RPMI- 1640 containing $10 \%$ FBS. All cells were kept in a $37^{\circ} \mathrm{C}$ humidified incubator with $5 \% \mathrm{CO}_{2}$. $\mathrm{HBMEC}$ was treated with indicated concentrations (from 0.1 to $1 \mu \mathrm{g} / \mathrm{ml}$ ) of recombinant ANXA2. OGD model was used to mimic ischemic stroke in vitro. The cells were cultured with glucose-free RPMI-1640 in a sealed chamber (Billups-Rothenberg, USA) loaded with mixed gas containing $95 \% \mathrm{~N}_{2}$ and5\% $\mathrm{CO}_{2}$ for 3 hrs. 3-(4,5-dimethylthiazol-2-yl)-2,5-diphenylte-trazolium bromide (MTT) assay was used to evaluate cell viability. Cell viability was expressed as a percentage relative to the normal cells. The data represented $6-12$ separate wells assayed per data point, with about $500-1000$ cells counted per well.

\section{In Vitro Angiogenesis Assays}

In vitro, angiogenesis was assessed by scratch migration and tube formation assays. The ability of endothelial cells to form capillary-like structures was evaluated by the Matrigel tube formation assay. In short, HBMECs $\left(4 \times 10^{4}\right.$ cells/well) were inoculated in 48-well culture plates which were pre-coated with growth factor-reduced Matrigel. Then cells were maintained with different treatments at $37^{\circ} \mathrm{C}$ for $24 \mathrm{hrs}$. The number of the branch point and tubes in 3 random fields from each well were counted.

To measure the migration abilities of HBMECs, scratch migration assay was performed. After being seeded in 12-well plates, HBMECs were scratched by a $200 \mu \mathrm{l}$ pipette tip. Then, the cells were incubated with different treatments for 2 days. HBMEC migration was monitored every day. Three different visual 
fields were randomly selected per well for observation and photograph under a phase contrast microscopy. The percentage of areas occupied by migrated cells were used to estimate the migration ability of HBMECs.

\section{Western blotting}

Cell or tissue samples were homogenized in RIPA buffer and the protein concentration was measured using a BCA assay. Samples of the same weight (50 $\mu \mathrm{g}$ per sample) were loaded on $12 \%$ SDS-PAGE gel, followed by transfer to PVDF membrane (Millipore, USA). The PVDF membranes were probed with the following primary antibodies: Tubulin (1: 5000), A2R (1: 1000), AKT (1: 1000), p-AKT (1: 1000), ERK (1: $1000)$ and p-ERK (1: 1000) at $4{ }^{\circ} \mathrm{C}$ overnight. HRP-linked secondary antibodies were then applied accordingly at room temperature for $2 \mathrm{hrs}$. Chemiluminescence was used to detect the signals of protein.

\section{Real-time PCR}

Real-time PCR was performed with MonAmp ${ }^{\mathrm{TM}}$ SYBR® Green qPCR Mix (MQ10101S, Monad, Shanghai), using a CFX-96 real-time PCR Detection system (Bio-Rad, USA). The relative quantities of total CDNA of ANXA $2^{+/+}$mice and wild-type mice were quantified using the $\triangle \triangle C T$ method. The primer sequences used were as follows: mouse Anxa2 (Fw: 5'- ATGTCTACTGTCCACGAAATCCT -3'; Rv: 5'CGAAGTTGGTGTAGGGTTTGACT -3'), mouse Actb (Fw: 5'- GGCTGTATTCCCCTCCATCG -3'; Rv: 5'CCAGTTGGTAACAATGCCATGT -3').

\section{Statistical Analysis}

All statistical data are represented as mean \pm s.e. All experiments were reproduced three - four independent times. For experiments with only two groups, unpaired two-tailed t-tests were used. For multiple comparisons, one-way or two-way ANOVA (with repeated measures when appropriate) followed by Tukey and Bonfferroni corrections were used. Data were considered statistically significant when the $p$ values were less than 0.05 .

\section{Result}

\section{Increased angiogenesis in $A N X A 2^{+/+}$mice}

Several in-vivo studies have demonstrated ANXA2-deficient mice displayed impaired neovascularization $[18,19]$. To determine the role of ANXA2 in angiogenesis, Anxa2 knock-in $\left(A N X A 2^{+/+}\right)$ mice were used. Anxa2 expression was increased in the cerebral cortex of $A N X A 2^{+/+}$mice

(Supplementary Fig. 1C). And western blotting showed that ANXA2 level was upregulated in the cerebral cortex of $A N X A 2^{+/+}$mice (Supplementary Fig. 1D, 1E). Next, to assess neovascularization in brain of $A N X A 2^{+/+}$mice, we dissected cortex from WT mice and $A N X A 2^{+/+}$mice, and measured density of lectin

(Fig. 1A). Our data confirmed that $A N X A 2^{+/+}$mice appeared to have increased density of microvessels

(Fig. 1B). We therefore investigated whether ANXA2 also directly affect endothelial cell proliferation. 
Aortic ring explants from WT mice and $A N X A 2^{+/+}$mice formed robust vessel structures (Fig. 1C). Compared with that of WT explants, the number of vessels emanating from $A N X A 2^{+/+}$mice explants was increased by about 3 folds (Fig. 1D). Taken together, these data indicated an increased angiogenesis in $A N X A 2^{+/+}$mice.

\section{ANXA2 promotes long-term neurological recovery after ischemic stroke}

To assess whether increased angiogenesis in $A N X A 2^{+/+}$mice could affects long-term neurological outcome after stroke, we carried out experiments using a Photothrombotic model of stroke. Mice were treated with BrdU before the onset of stroke. As expected, the number of BrdU positive and lectin positive cells was significantly increased in $A N X A 2^{+/+}$mice compared to WT mice (Fig. 2A, 2B). Early recanalization of blood vessels is correlated with better outcome by restoration of blood supply in ischemic zone, and reduction of cerebral infarction area [20-22]. We next applied Grid-walking and cylinder tests to comprehensively evaluate the sensorimotor functions after stroke. Compared with WT mice, $A N X A 2^{+/+}$mice exhibited significant sensorimotor functional improvements in all the behavioral tests, and these sensorimotor improvements were most prominent in the day 7 and day 14 after stroke (Fig. 2C, 2D). Taken together, these data suggest that ANXA2 enhances the overall long-term neurological recovery after stroke.

\section{Recombinant ANXA2 promotes in vitro angiogenesis}

We next asked whether recombinant ANXA2 promotes in vitro angiogenesis in culture of human brain microvascular endothelial cells (HBMEC) using scratch assay and capillary tube formation assay. Treatments of recombinant ANXA2 did not affect cell viability (Fig. 3A). In vitro scratch assay demonstrated that recombinant ANXA2 significantly increased HBMEC migration at 48 hours (Fig. 3B, 3C). Moreover, capillary tube formation assay revealed that recombinant ANXA2 significantly increased

the tube formation (Fig. 3D, 3E), showing elevated branch points (Fig. 3F). Thus, recombinant ANXA2 promotes in vitro angiogenesis.

\section{Recombinant ANXA2 rescues OGD-inhibited HBMEC angiogenesis}

We further examined the effects of recombinant ANXA2 on proangiogenic activities after OGD injury. To determine whether recombinant ANXA2 protects HBMEC from OGD-induced cytotoxicity, HBMEC were treated with indicated dose of recombinant ANXA2 treatment during OGD injury. Our result showed that $1 \mu \mathrm{g} / \mathrm{ml}$ recombinant ANXA2 lead to statistically significant increase HBMEC viability compared to OGD alone group (Fig. 4A). In vitro scratch and capillary tube formation assays showed that OGD injury induced significant decrease endothelial cells migration and tube formation (Fig. 4B-4F), whereas recombinant ANXA2 reversed these effects (Fig. 4B-4F). Collectively, these results demonstrate that the recombinant ANXA2 rescues OGD-inhibited angiogenic activities in HBMEC.

\section{ANXA2-induced angiogenesis is ANXA2 receptor dependent}


ANXA2 receptor (A2R) presenting on the cell surface has been found as a new vascular regulatory molecule [23]. In cancer cells, A2R can interact with ANXA2 to promote cell adhesion, migration and proliferation $[24,25]$. To elucidate whether A2R is involved ANXA2-induced angiogenesis, we performed Western blotting to check A2R expression after recombinant ANXA2 treatment. Recombinant ANXA2 led to significantly increased expression of A2R (Fig. 5A). More importantly, immunoprecipitation experiments from HBMEC cultures demonstrated that A2R was indeed able to bind to ANXA2 (Fig. 5B). We next examined the role of $A 2 R$ in the ANXA2-induced angiogenesis. Endogenous expression of $A 2 R$ was suppressed by siRNA (Fig. 5C). Compared to scrambled siRNA control, suppression of endogenous A2R diminished recombinant ANXA2-induced endothelial cells migration (Fig. 5D, 5E). We also observed significant decrease of tube formation indicated by reduced branch points in A2R siRNA group when compared to scrambled siRNA control (Fig. 5F-5H). These data suggested that ANXA2-induced angiogenesis is $\mathrm{A} 2 \mathrm{R}$ dependent.

\section{ANXA2-induced AKT and ERK activation is ANXA2 receptor dependent}

The two major pathways that regulate endothelial cells proliferation and migration are the ERK and AKT pathways [26]. Activation of these pathways in endothelial cells is necessary for angiogenesis [27,28]. To confirm recombinant ANXA2 could active ERK and AKT pathways, Western blotting was performed to detect AKT and ERK phosphorylation (Fig. 6A). We found recombinant ANXA2 successfully activated both pathways (Fig. 6B). Interestingly, compared with scrambled siRNA control, recombinant ANXA2 failed to activate both ERK and AKT when endogenous A2R was suppressed (Fig. 6C, 6D). Taken together, these results suggest that ANXA2-induced AKT and ERK activation is A2R dependent.

\section{Discussion}

\section{Acknowledgements}

Not applicable.

\section{Author Contributions}

WL and HD designed the study. WL and HL performed the experiments. WL and HL analyzed the data. $H D, W L, Z Y$ and $H L$ wrote the paper. All authors read and approved the final manuscript.

\section{Funding}

This work was supported by the foundations from National Natural Science Foundation of China (81703498 to WL; 82173789,81773700 and 81573402 to HD), Natural Science Foundation of Zhejiang Province (Y19H090117, YY19H310010 and LY16H310001), and Zhejiang Medical Health Science and Technology Plan (2018RC036).

\section{Data Availability}


All raw data and materials are available upon reasonable request to Haibin Dai.

\section{Compliance with Ethical Standards}

\section{Conflict of interest}

The author declare that they have no conflict of interest.

\section{Ethical Approval}

All protocols were performed in accordance with the Zhejiang University Guide for the Care and Use of Laboratory Animals

\section{Consent to Participate}

Not applicable

\section{Consent for Publication}

All authors have read the manuscript and agreed for its publication.

\section{Abbreviations}

ANXA2 Annexin A2

WT wild type

$A N X A 2^{+/+}$Anxa2 knock-in

A2R ANXA2 receptor

I/R ischemia/reperfusion

OIR oxygen-induced retinopathy

\section{Declarations}

\section{Declarations}

\section{Conflict of interest}

The author declare that they have no conflict of interest.

\section{Ethical Approval}


All protocols were performed in accordance with the Zhejiang University Guide for the Care and Use of Laboratory Animals

\section{Consent to Participate}

Not applicable

\section{Consent for Publication}

All authors have read the manuscript and agreed for its publication.

\section{Funding}

This work was supported by the foundations from National Natural Science Foundation of China (81703498 to WL; 82173789,81773700 and 81573402 to HD), Natural Science Foundation of Zhejiang Province (Y19H090117, YY19H310010 and LY16H310001), and Zhejiang Medical Health Science and Technology Plan (2018RC036).

\section{Author Contributions}

WL and HD designed the study. WL and HL performed the experiments. WL and $H L$ analyzed the data. $H D, W L, Z Y$ and $H L$ wrote the paper. All authors read and approved the final manuscript.

\section{Acknowledgements}

Not applicable.

\section{References}

1. Writing Group M, Lloyd-Jones D, Adams RJ, Brown TM, Carnethon M, Dai S, De Simone G, Ferguson TB, Ford E, Furie K, Gillespie C, Go A, Greenlund K, Haase N, Hailpern S, Ho PM, Howard V, Kissela B, Kittner S, Lackland D, Lisabeth L, Marelli A, McDermott MM, Meigs J, Mozaffarian D, Mussolino M, Nichol G, Roger VL, Rosamond W, Sacco R, Sorlie P, Roger VL, Thom T, Wasserthiel-Smoller S, Wong ND, Wylie-Rosett J, American Heart Association Statistics C, Stroke Statistics S (2010) Heart disease and stroke statistics-2010 update: a report from the American Heart Association. Circulation 121(7):e46-e215. doi:10.1161/CIRCULATIONAHA.109.192667

2. Lopez AD, Mathers CD, Ezzati M, Jamison DT, Murray CJ (2006) Global and regional burden of disease and risk factors, 2001: systematic analysis of population health data. Lancet 367(9524):1747-1757. doi:10.1016/S0140-6736(06)68770-9 
3. Manuel GE, Johnson T, Liu D (2017) Therapeutic angiogenesis of exosomes for ischemic stroke. Int J Physiol Pathophysiol Pharmacol 9(6):188-191

4. Ucuzian AA, Gassman AA, East AT, Greisler HP (2010) Molecular mediators of angiogenesis. J Burn Care Res 31(1):158-175. doi:10.1097/BCR.0b013e3181c7ed82

5. Li W, Chen Z, Yuan J, Yu Z, Cheng C, Zhao Q, Huang L, Hajjar KA, Chen Z, Lo EH, Dai H, Wang X (2019) Annexin A2 is a Robo4 ligand that modulates ARF6 activation-associated cerebral transendothelial permeability. J Cereb Blood Flow Metab 39(10):2048-2060. doi:10.1177/0271678X18777916

6. Sharma MC, Tuszynski GP, Blackman MR, Sharma M (2016) Long-term efficacy and downstream mechanism of anti-annexinA2 monoclonal antibody (anti-ANX A2 mAb) in a pre-clinical model of aggressive human breast cancer. Cancer Lett 373(1):27-35. doi:10.1016/j.canlet.2016.01.013

7. Swisher JF, Burton N, Bacot SM, Vogel SN, Feldman GM (2010) Annexin A2 tetramer activates human and murine macrophages through TLR4. Blood 115(3):549-558. doi:10.1182/blood-2009-06226944

8. Dallacasagrande V, Hajjar KA (2020) Annexin A2 in Inflammation and Host Defense. Cells 9 (6). doi:10.3390/cells9061499

9. Zhang S, Yu M, Guo Q, Li R, Li G, Tan S, Li X, Wei Y, Wu M (2015) Annexin A2 binds to endosomes and negatively regulates TLR4-triggered inflammatory responses via the TRAM-TRIF pathway. Sci Rep 5:15859. doi:10.1038/srep15859

10. Huang B, Deora AB, He KL, Chen K, Sui G, Jacovina AT, Almeida D, Hong P, Burgman P, Hajjar KA (2011) Hypoxia-inducible factor-1 drives annexin A2 system-mediated perivascular fibrin clearance in oxygen-induced retinopathy in mice. Blood 118(10):2918-2929. doi:10.1182/blood-2011-03-341214

11. Wang X, Fan X, Yu Z, Liao Z, Zhao J, Mandeville E, Guo S, Lo EH, Wang X (2014) Effects of tissue plasminogen activator and annexin A2 combination therapy on long-term neurological outcomes of rat focal embolic stroke. Stroke 45(2):619-622. doi:10.1161/STROKEAHA.113.003823

12. Nicosia RF, Ottinetti $A$ (1990) Growth of microvessels in serum-free matrix culture of rat aorta. A quantitative assay of angiogenesis in vitro. Lab Invest 63(1):115-122

13. Lee JK, Kim JE, Sivula M, Strittmatter SM (2004) Nogo receptor antagonism promotes stroke recovery by enhancing axonal plasticity. The Journal of neuroscience: the official journal of the Society for Neuroscience 24(27):6209-6217. doi:10.1523/jneurosci.1643-04.2004

14. Clarkson AN, Huang BS, Macisaac SE, Mody I, Carmichael ST (2010) Reducing excessive GABAmediated tonic inhibition promotes functional recovery after stroke. Nature 468(7321):305-309. doi:10.1038/nature09511

15. Lin YH, Dong J, Tang Y, Ni HY, Zhang Y, Su P, Liang HY, Yao MC, Yuan HJ, Wang DL, Chang L, Wu HY, Luo CX, Zhu DY (2017) Opening a New Time Window for Treatment of Stroke by Targeting HDAC2. The Journal of neuroscience: the official journal of the Society for Neuroscience 37(28):6712-6728. doi:10.1523/jneurosci.0341-17.2017 
16. Baskin YK, Dietrich WD, Green EJ (2003) Two effective behavioral tasks for evaluating sensorimotor dysfunction following traumatic brain injury in mice. J Neurosci Methods 129(1):87-93. doi:10.1016/s0165-0270(03)00212-7

17. Li W, Chen Z, Yan M, He P, Chen Z, Dai H (2016) The protective role of isorhamnetin on human brain microvascular endothelial cells from cytotoxicity induced by methylglyoxal and oxygen-glucose deprivation. J Neurochem 136(3):651-659. doi:10.1111/jnc.13436

18. Ling Q, Jacovina AT, Deora A, Febbraio M, Simantov R, Silverstein RL, Hempstead B, Mark WH, Hajjar KA (2004) Annexin II regulates fibrin homeostasis and neoangiogenesis in vivo. J Clin Invest 113(1):38-48. doi:10.1172/JCl19684

19. Jacovina AT, Deora AB, Ling Q, Broekman MJ, Almeida D, Greenberg CB, Marcus AJ, Smith JD, Hajjar KA (2009) Homocysteine inhibits neoangiogenesis in mice through blockade of annexin A2dependent fibrinolysis. J Clin Invest 119(11):3384-3394. doi:10.1172/JCI39591

20. Arai K, Jin G, Navaratna D, Lo EH (2009) Brain angiogenesis in developmental and pathological processes: neurovascular injury and angiogenic recovery after stroke. FEBS J 276(17):4644-4652. doi:10.1111/j.1742-4658.2009.07176.x

21. Zhang ZG, Chopp M (2009) Neurorestorative therapies for stroke: underlying mechanisms and translation to the clinic. Lancet Neurol 8(5):491-500. doi:10.1016/S1474-4422(09)70061-4

22. Krupinski J, Kaluza J, Kumar P, Kumar S, Wang JM (1994) Role of angiogenesis in patients with cerebral ischemic stroke. Stroke 25(9):1794-1798. doi:10.1161/01.str.25.9.1794

23. Shiozawa Y, Havens AM, Jung Y, Ziegler AM, Pedersen EA, Wang J, Wang J, Lu G, Roodman GD, Loberg RD, Pienta KJ, Taichman RS (2008) Annexin II/annexin II receptor axis regulates adhesion, migration, homing, and growth of prostate cancer. J Cell Biochem 105(2):370-380. doi:10.1002/jcb.21835

24. Mussunoor S, Murray GI (2008) The role of annexins in tumour development and progression. J Pathol 216(2):131-140. doi:10.1002/path.2400

25. Xiong Y, Fan C, Kong L, Dong L, Zhu N, Zhang J, Wang L, Qin T, Shen Y, Chen M (2013) Annexin II receptor induces apoptosis independent of Annexin II. Apoptosis 18(8):925-939. doi:10.1007/s10495-013-0846-2

26. Murphy DA, Makonnen S, Lassoued W, Feldman MD, Carter C, Lee WM (2006) Inhibition of tumor endothelial ERK activation, angiogenesis, and tumor growth by sorafenib (BAY43-9006). Am J Pathol 169(5):1875-1885. doi:10.2353/ajpath.2006.050711

27. Jiang BH, Zheng JZ, Aoki M, Vogt PK (2000) Phosphatidylinositol 3-kinase signaling mediates angiogenesis and expression of vascular endothelial growth factor in endothelial cells. Proc Natl Acad Sci U S A 97(4):1749-1753. doi:10.1073/pnas.040560897

28. Berra E, Milanini J, Richard DE, Le Gall M, Vinals F, Gothie E, Roux D, Pages G, Pouyssegur J (2000) Signaling angiogenesis via p42/p44 MAP kinase and hypoxia. Biochem Pharmacol 60(8):11711178. doi:10.1016/s0006-2952(00)00423-8 
29. Lamalice L, Le Boeuf F, Huot J (2007) Endothelial cell migration during angiogenesis. Circ Res 100(6):782-794. doi:10.1161/01.RES.0000259593.07661.1e

30. Hatakeyama M, Ninomiya I, Kanazawa M (2020) Angiogenesis and neuronal remodeling after ischemic stroke. Neural Regen Res 15(1):16-19. doi:10.4103/1673-5374.264442

31. Song H, Pan D, Sun W, Gu C, Zhang Y, Zhao P, Qi Z, Zhao S (2015) SiRNA directed against annexin II receptor inhibits angiogenesis via suppressing MMP2 and MMP9 expression. Cell Physiol Biochem 35(3):875-884. doi:10.1159/000369745

\section{Figures}

Figure 1

A
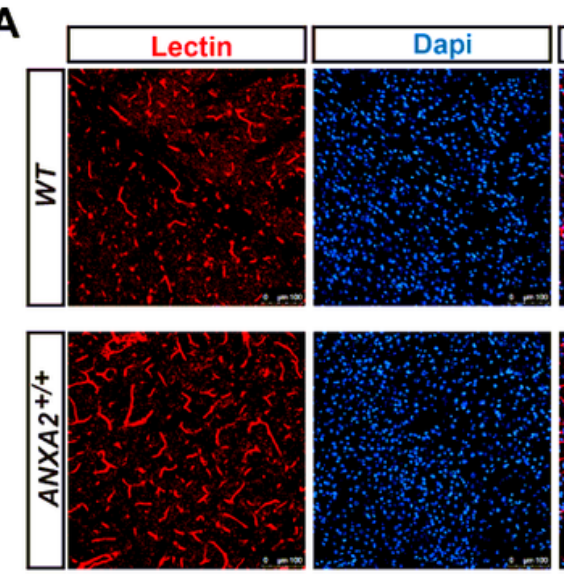

Dapi
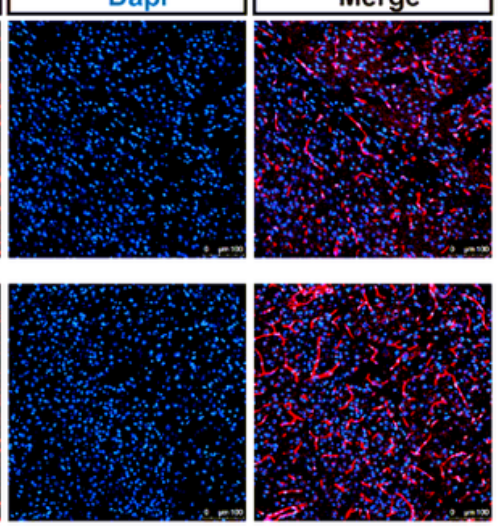

B

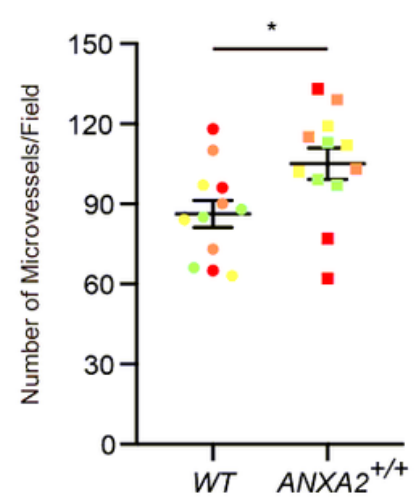

C
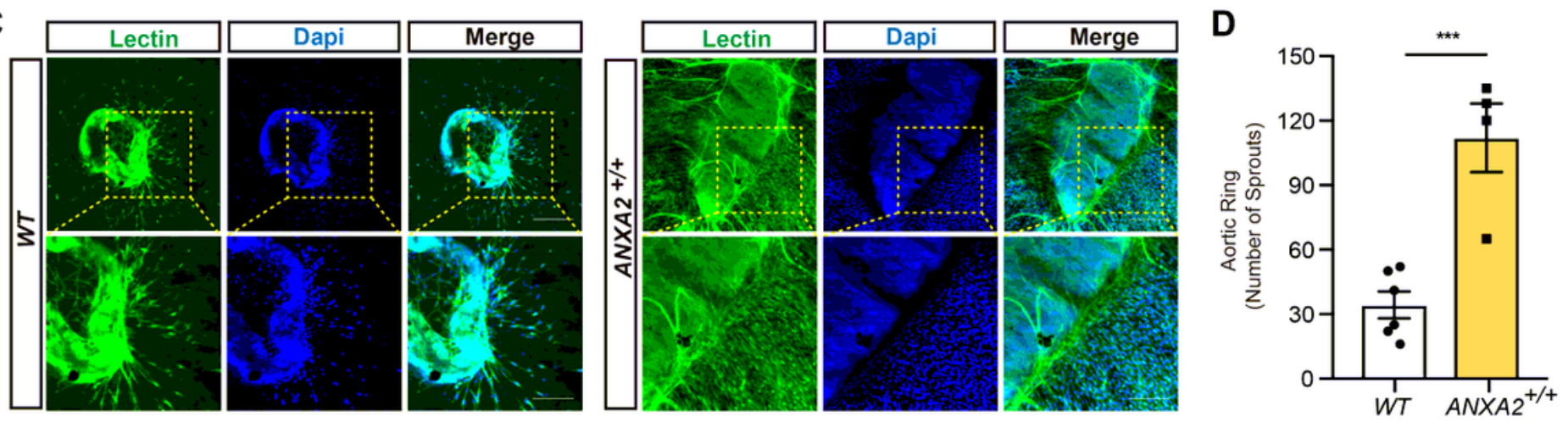

Figure 1

Increased angiogenesis in ANXA2+/+ mice. A, representative lectin and DAPI immunostaining in the cerebral cortex of WT mice and ANXA2+/+ mice. Scale bar, $140 \mu \mathrm{m}$. B, number of microvessels in the cerebral cortex of WT mice and ANXA2+/+ mice. C, representative lectin and DAPI immunostaining of aortic ring of WT mice and ANXA2+/+ mice. Scale bar, $300 \mu \mathrm{m}$ (top), $150 \mu \mathrm{m}$ (bottom). D, number of sprouts from aortic ring of WT mice and ANXA2+/+ mice at day 6 after culture ex vivo. Data are mean \pm s.e. ${ }^{*} \mathrm{P}<0.05 ; * \star \star P<0.001$. Two-tailed t-test. 
Figure 2

A

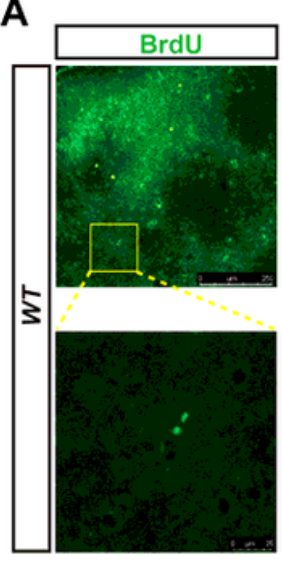

C

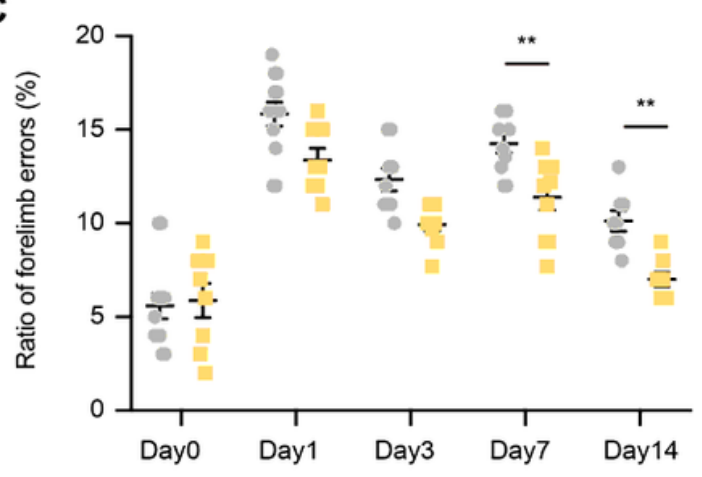

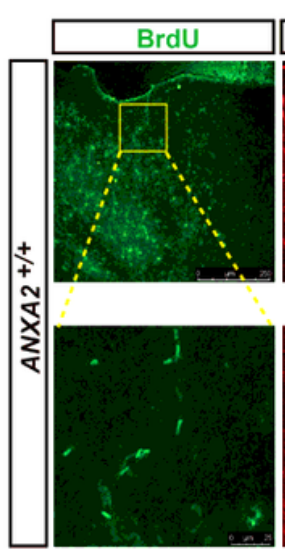

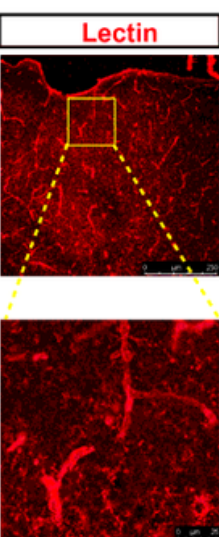

D

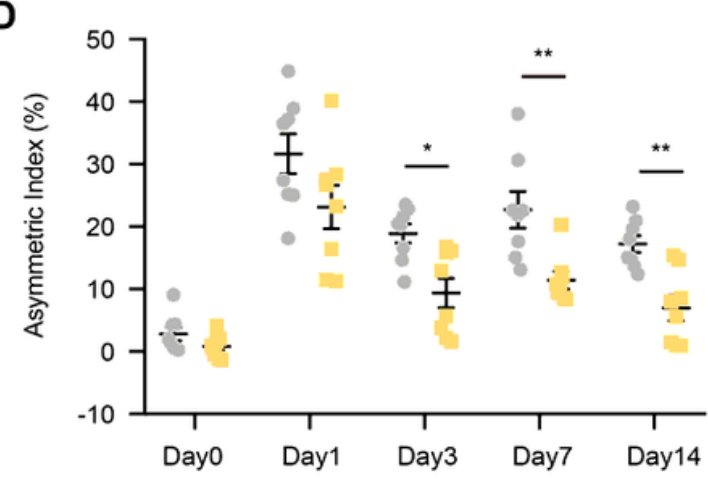

B
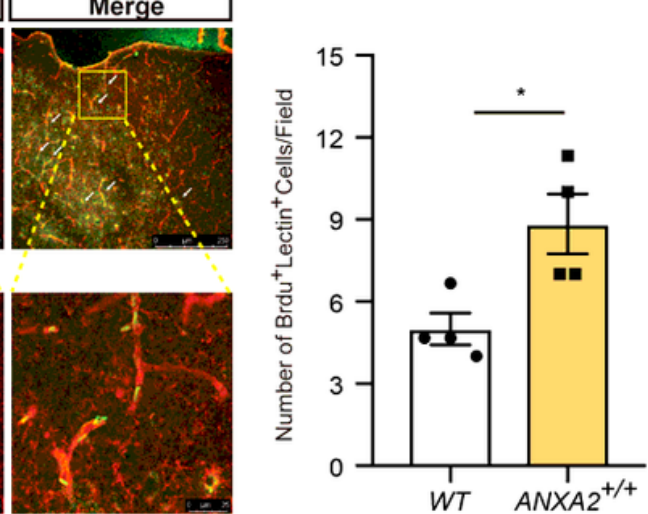

$\square$ WT

\section{Figure 2}

ANXA2 promotes long-term neurological recovery after ischemic stroke. A, representative lectin, BrdU and DAPI immunostaining of WT mice and ANXA2+/+ mice at day 7 after stroke. Scale bar, $250 \mu \mathrm{m}$ (top), 45 $\mu \mathrm{m}$ (bottom). B, number of lectin+ and BrdU+ cells of WT mice and ANXA2+/+ mice at day 7 after stroke. C, Foot faults of left forelimb in the grid-walking task. D, Forelimb symmetry in the cylinder task. Data are mean \pm s.e. ${ }^{*} \mathrm{P}<0.05 ;{ }^{*} \mathrm{P}<0.01$. Two-tailed t-test $(\mathrm{B})$; two-way ANOVA with Bonferroni adjustment (C, D). 
Figure 3

A

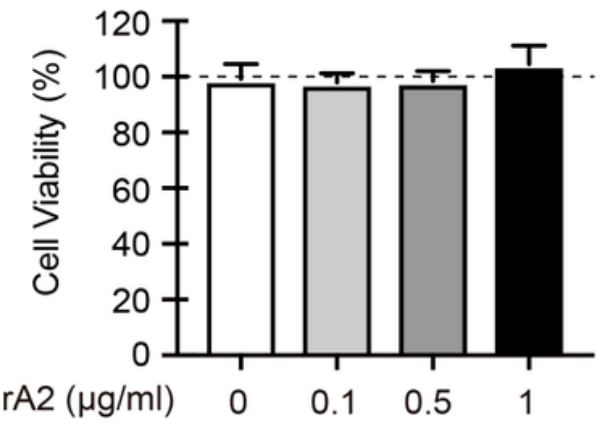

D
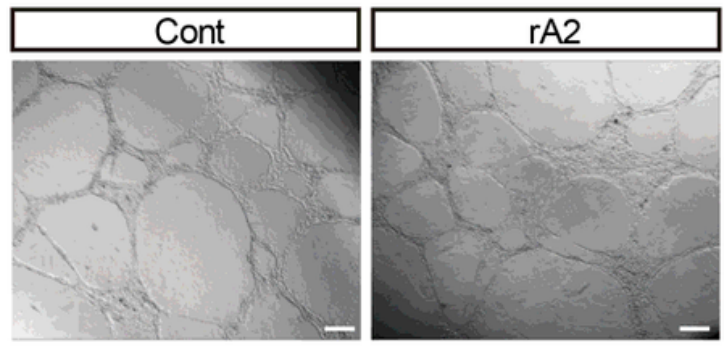

B
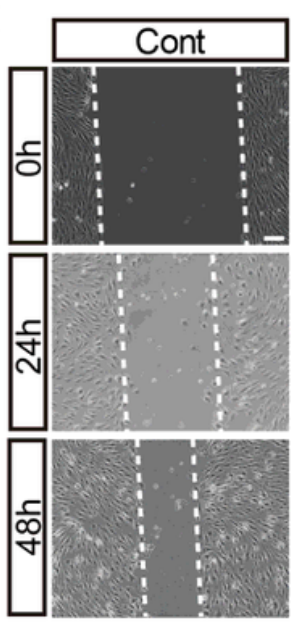

E
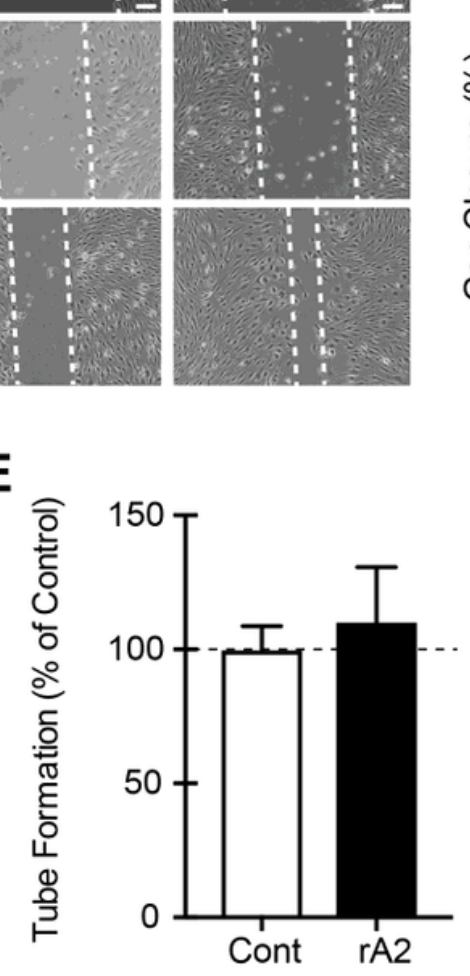

C
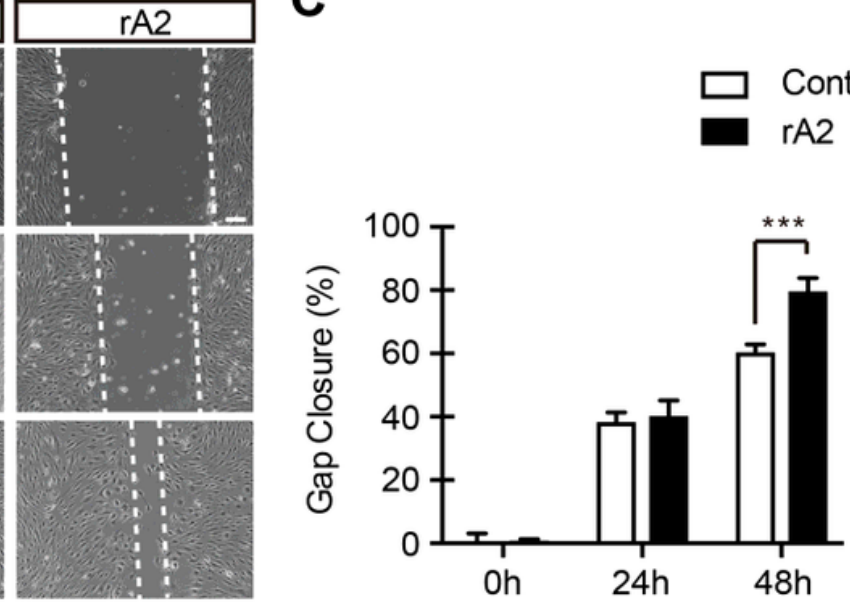

$\mathbf{F}$

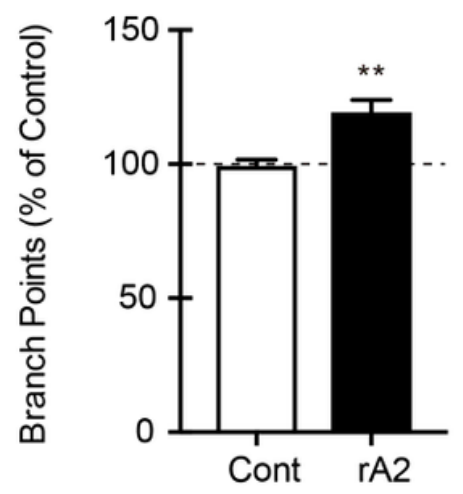

Figure 3

Recombinant ANXA2 promotes in vitro angiogenesis. A, effects of different dose of rA2 $(0.1 \mu \mathrm{g} / \mathrm{ml}$ $-1 \mu \mathrm{g} / \mathrm{ml})$ on the HBMEC viability. B-C, effects of $\mathrm{rA} 2(1 \mu \mathrm{g} / \mathrm{ml})$ on scratch migration in HBMEC (B) and bar graph of gap closure (C). D-F, effects of rA2 $(1 \mu \mathrm{g} / \mathrm{ml})$ on tube formation (D) and bar graph of the number of tubes (E) and the number of branch points (F). Scale bar, $100 \mu \mathrm{m} . \mathrm{n}=3$ independent experiments. Data are mean \pm s.e. ${ }^{\star \star} \mathrm{P}<0.01 ;{ }^{\star \star \star} \mathrm{P}<0.001$. Two-tailed $\mathrm{t}$-test $(\mathrm{F})$; two-way ANOVA with Bonferroni adjustment (C). 
Figure 4

A

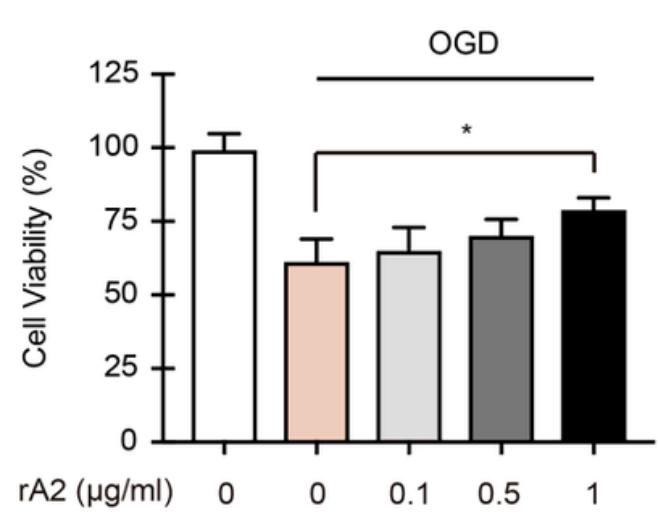

D

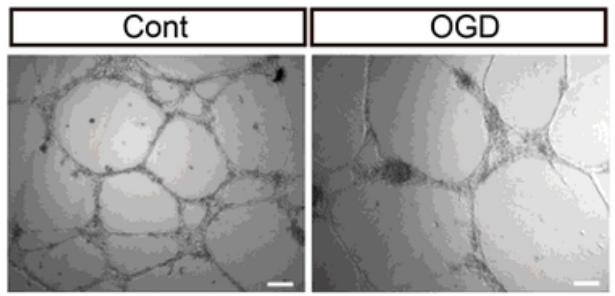

B

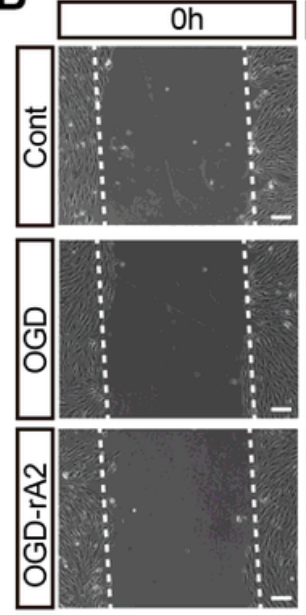

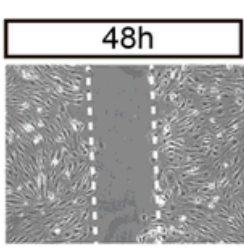

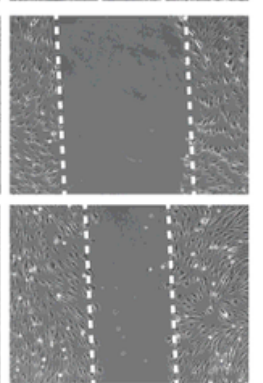

E

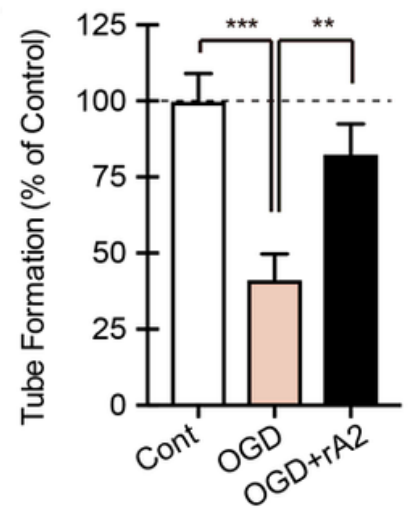

C

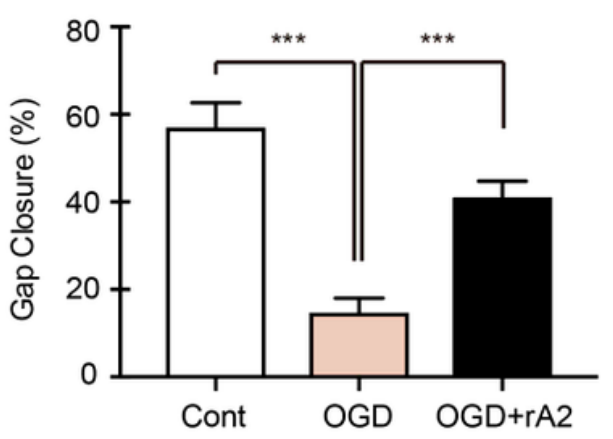

$\mathbf{F}$

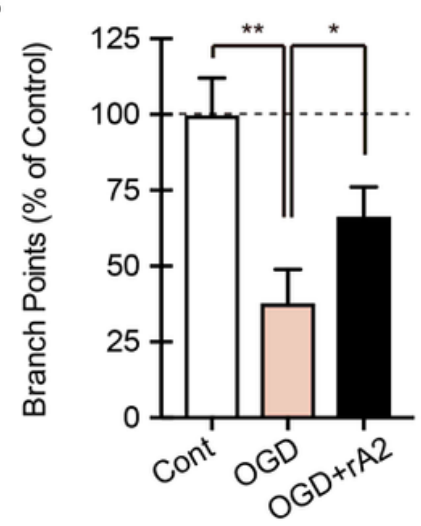

Figure 4

Recombinant ANXA2 rescued OGD-inhibited HBMEC angiogenesis. A, effects of different dose of rA2 $(0.1 \mu \mathrm{g} / \mathrm{ml}-1 \mu \mathrm{g} / \mathrm{ml})$ on the viability of HBMEC treated with $3 \mathrm{hrs}$ OGD and $21 \mathrm{hrs}$ reperfusion. B-C, effects of rA2 $(1 \mu \mathrm{g} / \mathrm{ml})$ on scratch migration in HBMEC treated with $3 \mathrm{hrs}$ OGD and $21 \mathrm{hrs}$ reperfusion (B) and bar graph of gap closure (C). D-F, effects of $\mathrm{rA2}(1 \mu \mathrm{g} / \mathrm{ml})$ on tube formation in HBMEC treated with 3 hrs OGD and $21 \mathrm{hrs}$ reperfusion (D) and bar graph of the number of tubes $(E)$ and the number of branch points $(F)$. Scale bar, $100 \mu \mathrm{m} . \mathrm{n}=3$ independent experiments. Data are mean \pm s.e. ${ }^{*} \mathrm{P}<0.05$; ${ }^{\star *} \mathrm{P}<0.01$; $\star \star * P<0.001$. One-way ANOVA with post-hoc Tukey adjustment. 
Figure 5

A
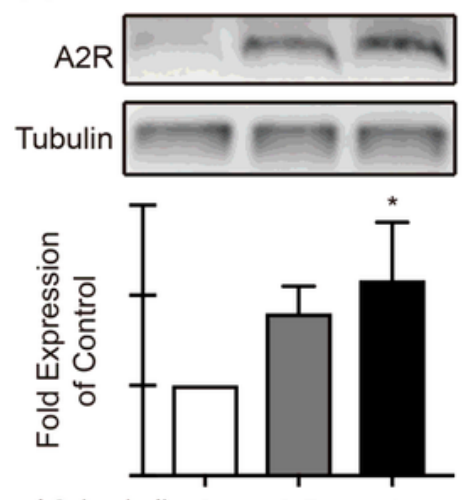

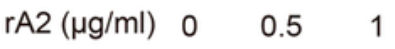

B

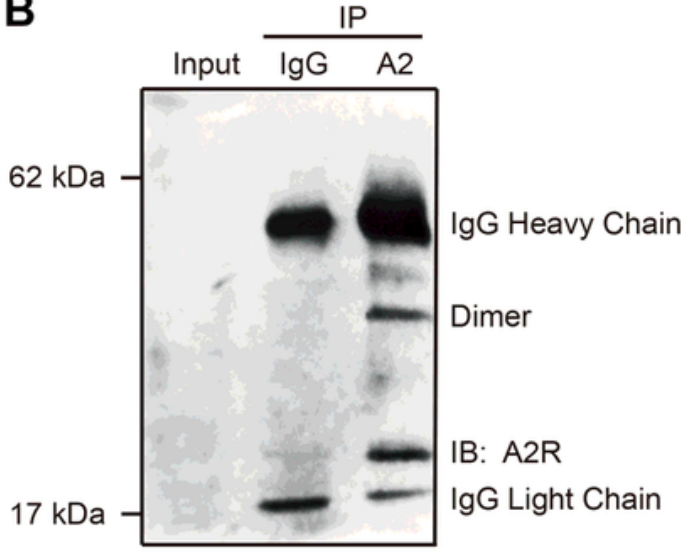

C

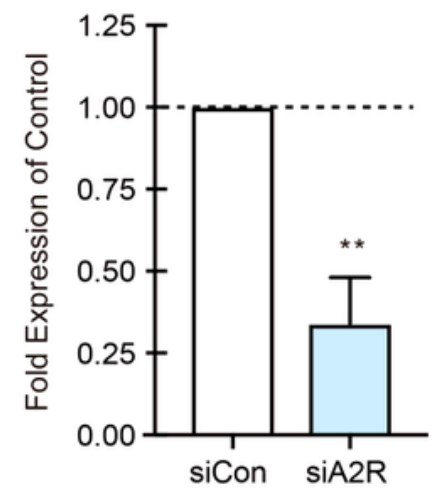

D
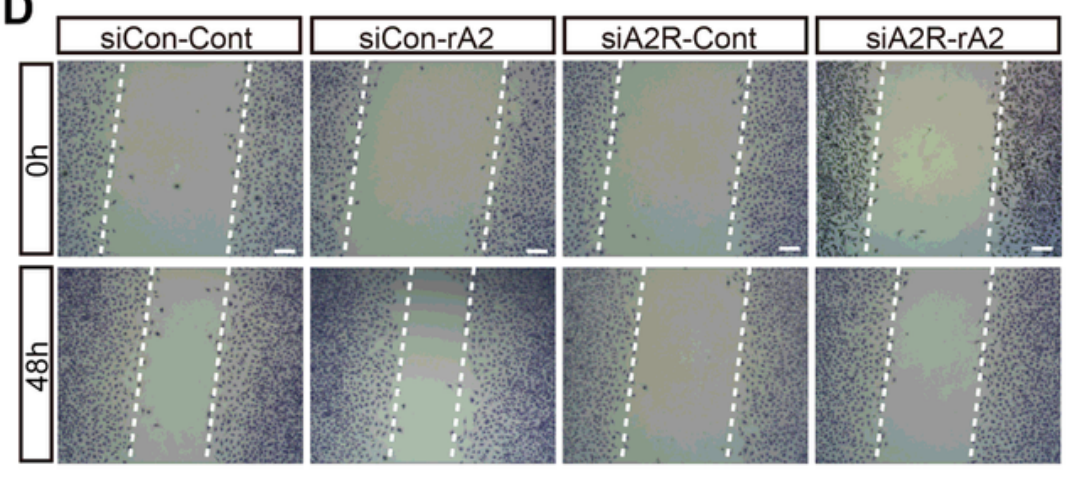

E

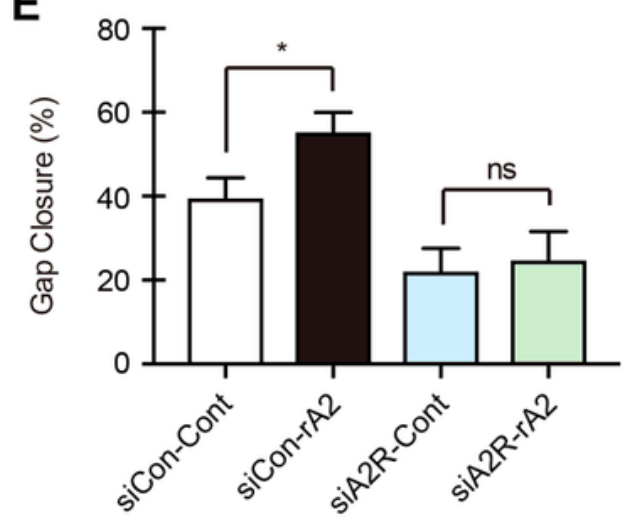

F

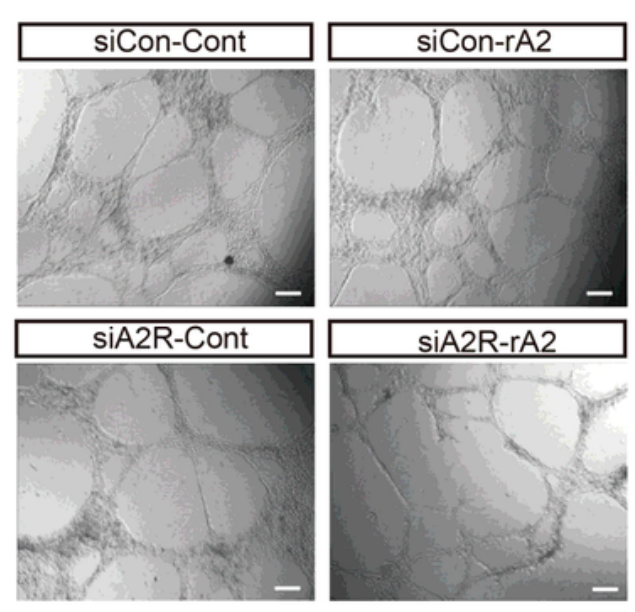

G

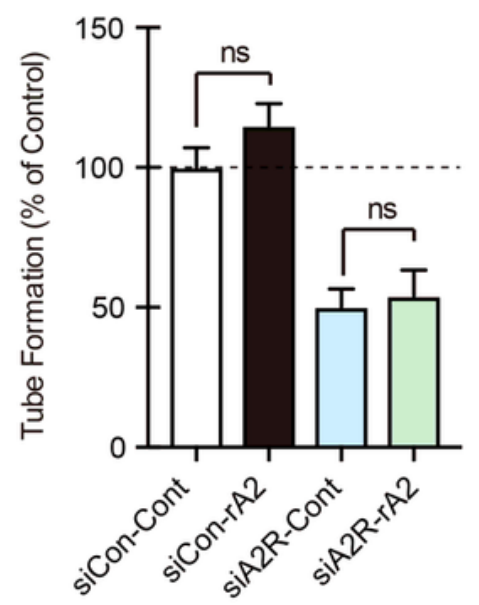

H

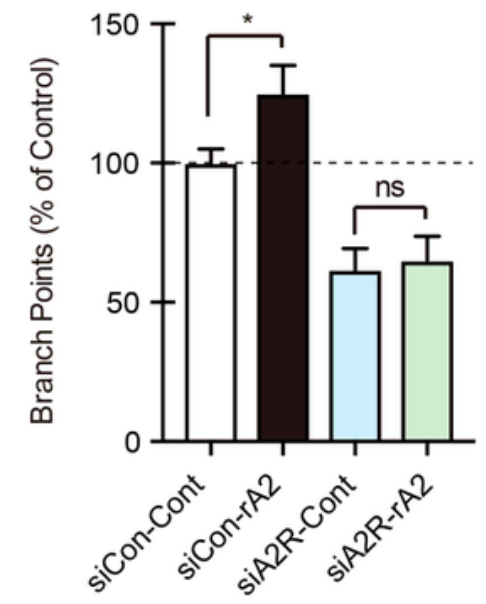

\section{Figure 5}

ANXA2-induced angiogenesis is ANXA2 receptor dependent. A, expression of A2R in rA2 $(0.5 \mu \mathrm{g} / \mathrm{ml}$ $-1 \mu \mathrm{g} / \mathrm{ml}$ ) treated HBMEC cultures. B, Immunoprecipitation (IP) of HBMEC with anti-ANXA2 (A2) followed by immunoblotting with anti-A2R. C, A2R RNA expression in A2R and control (Con) short interfering RNA (siRNA) transfected HBMEC cultures. D-E, effects of control siRNA and A2R siRNA on scratch migration in HBMEC treated with $48 \mathrm{hrs} \mathrm{rA2}(1 \mu \mathrm{g} / \mathrm{ml})(\mathrm{D})$ and bar graph of gap closure $(\mathrm{E})$. F-H, effects of control 
siRNA and A2R siRNA on tube formation in HBMEC treated with $48 \mathrm{hrs} \mathrm{rA2}(1 \mu \mathrm{g} / \mathrm{ml})(\mathrm{F})$ and bar graph of the number of tubes $(G)$ and the number of branch points $(H)$. Scale bar, $100 \mu \mathrm{m} . n=3$ independent experiments. Data are mean \pm s.e. ${ }^{*} \mathrm{P}<0.05 ;{ }^{* *} \mathrm{P}<0.01 ; * \star * \mathrm{P}<0.001$. Two-tailed t-test (C); One-way ANOVA with post-hoc Tukey adjustment $(\mathrm{E}, \mathrm{G}, \mathrm{H})$.

\section{Figure 6}

A

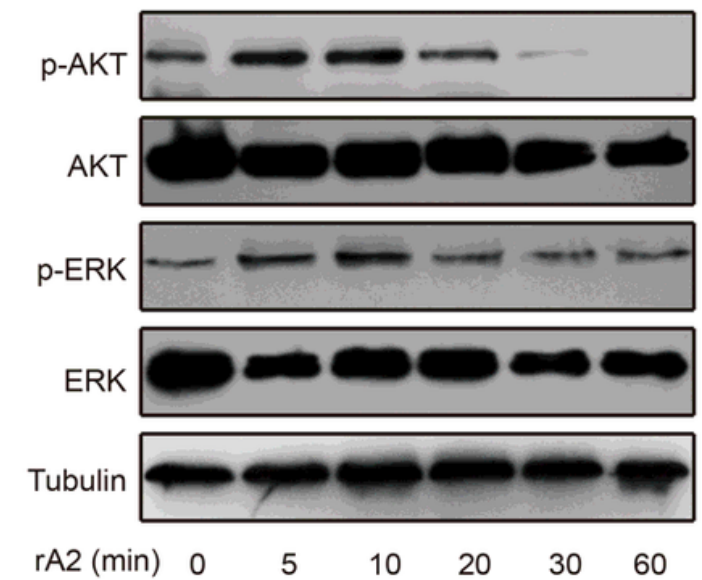

D

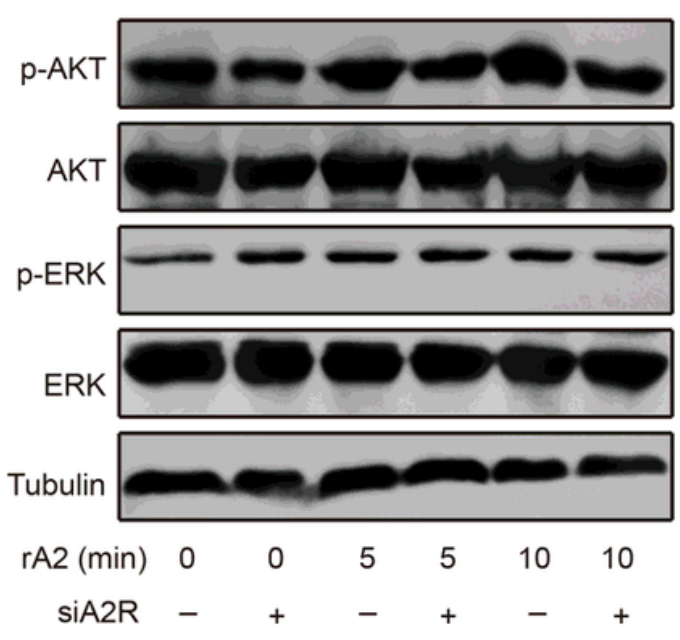

B

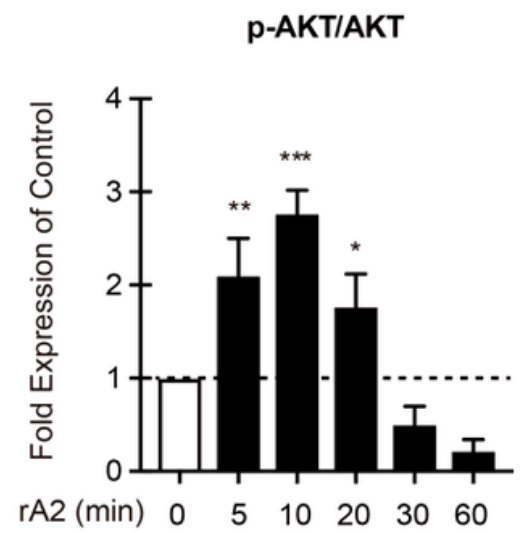

E

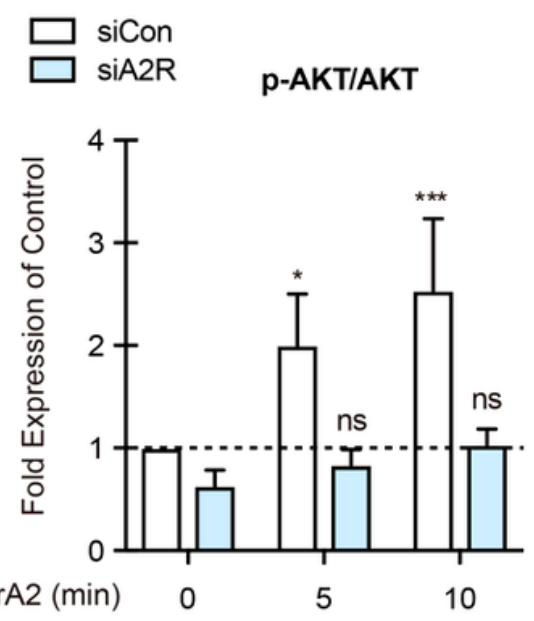

C

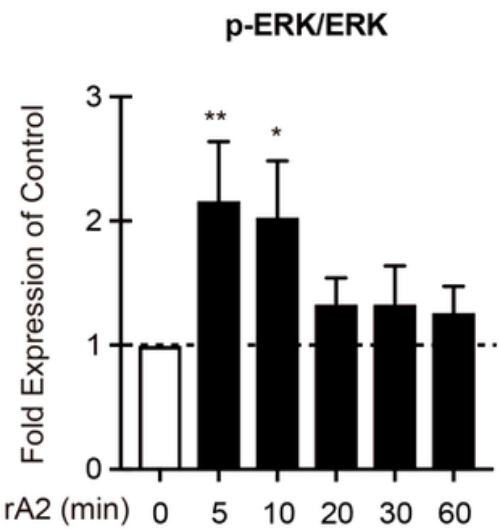

$\mathbf{F}$

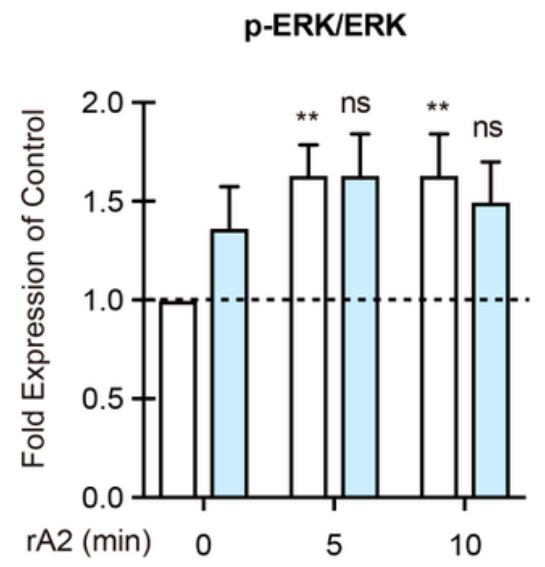

Figure 6

ANXA2-induced AKT and ERK activation is ANXA2 receptor dependent. A-C, pAKT/AKT and pERK/ERK expressions in HBMEC cultures treated with $r A 2(1 \mu \mathrm{g} / \mathrm{ml})$ at different time points. D-E, effects of control siRNA and A2R siRNA on pAKT/AKT and pERK/ERK expressions in HBMEC cultures treated with rA2 $(1 \mu \mathrm{g} / \mathrm{ml})$ at different time points. $\mathrm{n}=3$ independent experiments. Data are mean \pm s.e. ${ }^{*} \mathrm{P}<0.05 ; * \star \mathrm{P}<0.01$; ${ }^{\star * *} \mathrm{P}<0.001$. One-way ANOVA with post-hoc Tukey adjustment $(\mathrm{B}, \mathrm{C})$; two-way ANOVA with Bonferroni adjustment $(E, F)$.

\section{Supplementary Files}


This is a list of supplementary files associated with this preprint. Click to download.

- SupplementaryData.docx 